\title{
Elevated serum osteoprotegerin may predict peripheral arterial disease after kidney transplantation: A single-center prospective cross-sectional study in Taiwan
}

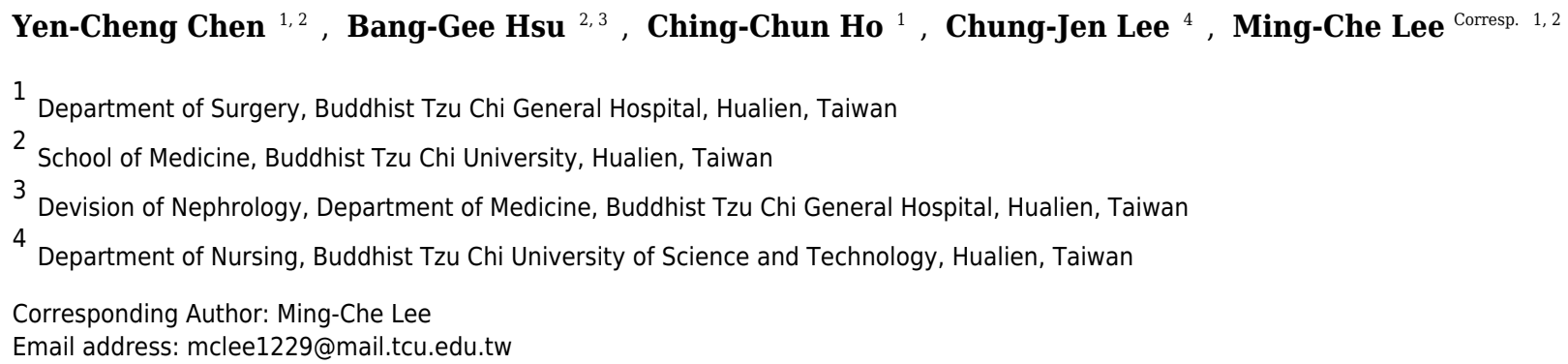

Background. Osteoprotegerin (OPG) is a potential biomarker for severity and complications of cardiovascular diseases. Peripheral arterial disease (PAD) is associated with an increased risk of death in kidney transplantation (KT) patients. This prospective cross-sectional study evaluated the relationship between serum OPG and PAD in KT patients.

Methods. Seventy-four KT patients were enrolled for this PAD study. Fasting blood samples were obtained to measure serum OPG levels by using enzyme-linked immunosorbent assay kits. The ankle-brachial index (ABI) of less than 0.9 was applied for PAD diagnosis.

Results. Thirteen patients (17.6\%) were diagnosed with PAD. Diabetes $(P=0.025)$, smoking $(P=0.010)$, and increased OPG levels $(P=0.001)$ were significantly more frequent in the PAD group. Multivariate logistic regression analysis showed that serum OPG (odds ratio [OR], 1.336; 95\% confidence interval [Cl], $1.108-1.611 ; \mathrm{P}=0.002)$ and diabetes $(\mathrm{OR}, 7.120 ; 95 \% \mathrm{Cl}, 1.080-46.940 ; \mathrm{P}=0.041)$ were independent predictors of PAD in KT patients. The area under the receiver operating characteristic (ROC) curve determined that the probability of a serum OPG level of $7.117 \mathrm{pg} / \mathrm{L}$ in predicting PAD in KT patients was $0.799(95 \% \mathrm{Cl}, 0.690-0.884 ; \mathrm{P}<0.001)$.

Discussion. Exploration of reliable biomarkers for early identification of vascular risk is crucial for KT patients. Elevated serum OPG levels may predict PAD in KT patients with cutoff value of $7.117 \mathrm{pg} / \mathrm{L}$. 


\section{Elevated Serum Osteoprotegerin May Predict Peripheral Arterial Disease}

2 After Kidney Transplantation: A Single-center Prospective Cross-sectional

\section{Study In Taiwan}

4 Yen-Cheng Chen ${ }^{1,2} *$, Bang-Gee $\mathrm{Hsu}^{2,3} *$, Ching-Chun $\mathrm{Ho}^{1}$, Chung-Jen Lee ${ }^{4}$, and Ming-Che 5 Lee $^{1,2}$

$6 \quad{ }^{1}$ Department of Surgery, Buddhist Tzu Chi General Hospital, Hualien, Taiwan

$7 \quad 2$ School of Medicine, Tzu Chi University, Hualien, Taiwan

$8{ }^{3}$ Division of Nephrology, Buddhist Tzu Chi General Hospital, Hualien, Taiwan

$9{ }^{4}$ Department of Nursing, Tzu Chi University of Science and Technology, Hualien, Taiwan

$11 *$ These authors contributed equally to this work.

13 Corresponding Author:

14 Ming-Che Lee, MD

15 No. 707, Section 3, Chung Yang Road, Hualien City, Hualien, 970, Taiwan

16 Email address: mingche1229@gmail.com 


\section{Abstract}

20 Background. Osteoprotegerin (OPG) is a potential biomarker for severity and complications of

21 cardiovascular diseases. Peripheral arterial disease (PAD) is associated with an increased risk of

22 death in kidney transplantation (KT) patients. This prospective cross-sectional study evaluated

23 the relationship between serum OPG and PAD in KT patients.

24 Methods. Seventy-four KT patients were enrolled for this PAD study. Fasting blood samples

25 were obtained to measure serum OPG levels by using enzyme-linked immunosorbent assay kits.

26 The ankle-brachial index (ABI) of less than 0.9 was applied for PAD diagnosis.

27 Results. Thirteen patients (17.6\%) were diagnosed with PAD. Diabetes $(P=0.025)$, smoking $(P$

$28=0.010)$, and increased OPG levels $(P=0.001)$ were significantly more frequent in the PAD

29 group. Multivariate logistic regression analysis showed that serum OPG (odds ratio [OR], 1.336;

$3095 \%$ confidence interval $[\mathrm{CI}], 1.108-1.611 ; P=0.002)$ and diabetes $(\mathrm{OR}, 7.120 ; 95 \% \mathrm{CI}, 1.080$

$3146.940 ; P=0.041)$ were independent predictors of PAD in KT patients. The area under the

32 receiver operating characteristic (ROC) curve determined that the probability of a serum OPG

33 level of $7.117 \mathrm{pg} / \mathrm{L}$ in predicting PAD in KT patients was $0.799(95 \% \mathrm{CI}, 0.690-0.884 ; P<$

34 0.001).

35 Discussion. Exploration of reliable biomarkers for early identification of vascular risk is crucial

36 for KT patients. Elevated serum OPG levels may predict PAD in KT patients with cutoff value 37 of $7.117 \mathrm{pg} / \mathrm{L}$. 
40

41 Peripheral arterial disease (PAD) is a common manifestation of atherosclerotic vascular disease

42 that is associated with significant morbidity and mortality (Criqui \& Aboyans 2015).

43 Establishing PAD diagnosis in clinical practice can be easily achieved by utilizing ankle-brachial

44 index (ABI), which is a marker of atherosclerosis (Cooke \& Wilson 2010). Vascular

45 calcification, the main pathological event underlying PAD, is a complicated process that involves

46 a shift in the phenotype of vascular smooth muscle cells to chondrocyte-like or osteoblast-like

47 cells, which ultimately leads to ectopic mineralization (Evrard et al. 2015). Osteoprotegerin

48 (OPG) is a cytokine belonging to the tumor necrosis factor (TNF) receptor superfamily and is an

important pathological mediator of arterial calcification as part of the OPG/receptor activator of nuclear factor-kB (RANK)/RANK ligand (RANKL) pathway (Venuraju et al. 2010). Several studies showed that a decrease in ABI is associated with increased risks of stroke, cardiovascular disease, and all-cause mortality (Doobay \& Anand 2005).

PAD is a relatively common manifestation in patients with chronic kidney disease and those undergoing kidney transplantation (KT). Serum OPG had been associated with the progression of PAD in KT patients (Ye et al. 2013). In the Assessment of LEscol in the Renal Transplantation (ALERT) study, elevated serum OPG levels were independently associated with the deterioration of renal function, cardiovascular events, and all-cause mortality in KT patients (Svensson et al. 2012). The purpose of the current study was to determine the relationship between serum OPG level and PAD, as determined by ABI, in KT patients.

\section{Material and Methods}

\section{Study design and Participants}


63 From April 2013 to June 2013, eighty-one KT patients were treated consecutively at a tertiary

64 medical center in Hualien, Taiwan. We excluded the patients with any acute infection, episodes

65 of rejection, proved malignancy, acute coronary syndrome or congestive heart failure status, and

66 the remained arteriovenous shunt over the limb during the 3-month study period. Besides, the

67 patients who take medications related to calcium, active vitamin D metabolites, estrogen,

68 bisphosphonates, or teriparatide were also excluded. Finally, seventy-four KT patients, including

6940 males and 34 females with ages ranging from 31 and 73 years were enrolled in this study for

70 further data collection and analyses. The study was conducted in accordance with the Declaration

71 of Helsinki and was approved by the local ethics committee of the institute (REC No.: IRB101-

72 144). Informed consent was obtained from all patients prior to their enrollment in this study.

73 Clinical parameters and Ankle-brachial index measurement

74 We recorded the lifestyle variables, medical conditions and relevant medications use. The body

75 weight, body height, waist circumference and body mass index were measured by trained

76 personnel as previously described (Lee et al. 2014). The ABI of this study was calculated by

77 using an ABI-form device (VaSera VS-1000, Fukuda Denshi, Tokyo, Japan) that measures blood

78 pressure in both upper arms and both ankles by an oscillometric method automatically. Under

79 supine position, the occlusion and monitoring cuffs were appropriately applied to all four limbs

80 of patient, and the real-time electrocardiography was recorded for at fifteen minutes at least. The

$81 \mathrm{ABI}$ is the ratio of the systolic blood pressure of the ankle divided by that of the arm, and the

82 lower systolic blood pressure of ankle was chosen to calculate ABI. As previous our study we set

830.9 as the cutoff value for PAD diagnosis (Lee et al. 2014).

84 Biochemical determinations

85 Fasting blood samples were obtained, and parts of samples centrifuged at $3000 \times \mathrm{g}$ for $10 \mathrm{~min}$ 
86 after collection for measuring complete blood cell count (Sysmex K-1000; Bohemia, NY, USA).

87 The other serum samples were immediately stored at $4{ }^{\circ} \mathrm{C}$ for biochemical analyses within one

88 hour after collection. Serum levels of blood urea nitrogen(BUN), creatinine (Cre), total

89 cholesterol (TCH), triglycerides (TG), high-density lipoprotein cholesterol(HDL-C), low-density

90 lipoprotein cholesterol (LDL-C), fasting glucose, calcium, and phosphorus were measured using

91 an autoanalyzer (COBAS Integra 800; Roche Diagnostics, Basel, Switzerland). The 4-variable

92 Modification of Diet in Renal Disease (MDRD) equation was applied for glomerular filtration

93 rate (eGFR) estimation in the study (Levey et al. 2006). Serum concentrations of OPG

94 (eBioscience; San Diego, CA, USA) and intact parathyroid hormone (Diagnostic Systems

95 Laboratories; Webster, TX, USA) were determined by commercial enzyme-linked

96 immunosorbent assay kits (Hsu et al. 2015; Wang et al. 2014; Lee et al. 2015). The limited of

97 detection, calculated as the concentration of human OPG and PTH levels corresponding to the

98 blank average minus three standard deviations, was $2.5 \mathrm{pg} / \mathrm{mL}$ and $1.57 \mathrm{pg} / \mathrm{mL}$, respectively. The

99 inter- and intra-assay coefficients of variation for OPG were 8.0\% and 7.0\%, and for iPTH were

$100 \quad 3.6 \%$ and $6.0 \%$.

\section{Statistical analysis}

102 Data were tested for normal distribution using the Kolmogorov-Smirnov test. Normally

103 distributed data were expressed as means with standard deviation, and comparisons between

104 patient groups were performed using the Student's independent $t$-test (two tailed). Data that were

105 not normally distributed were expressed as medians with interquartile ranges, and comparisons

106 of parameters (fasting glucose, blood urea nitrogen, creatinine, triglyceride, intact parathyroid

107 hormone, and OPG) between patients were performed by using Mann-Whitney U test. Data

108 expressed as the number of patients were analyzed with the chi-square test. The variables that 
109 showed the significant association with PAD were tested for independence by multivariate

110 logistic regression analysis. A receiver operating characteristic (ROC) curve was used to

111 calculate the area under the ROC curve (AUC) to identify the cutoff value of serum OPG value

112 for PAD prediction in KT patients. Data were analyzed using SPSS for Windows (version 19.0;

113 SPSS, Chicago, IL, USA). $P$ values less than 0.05 were identified as statistical significance.

114

\section{Results}

116 Among a total of $74 \mathrm{KT}$ patients that were enrolled, 40 were males, mean age was $52.07 \pm 9.63$

117 years, and mean post-transplantation duration was $72.19 \pm 42.99$ months. Tables 1 and 2 provide

118 the demographic and clinical characteristics, biochemical data, and comorbidities of all KT

119 patients enrolled in the study. In this cohort, $21(28.4 \%)$ and $36(48.6 \%)$ patients had diabetes

120 and hypertension, respectively. In addition, 5 patients $(6.8 \%)$ were smokers, whereas none of the

121 patients had a history of stroke.

122 Thirteen patients (17.6\%) were determined to have PAD, with a mean ABI of 0.88 , whereas the

123 mean $\mathrm{ABI}$ of the remaining 61 patients without PAD was $1.10(P<0.001)$. There were

124 significantly more patients with diabetes $(P=0.025)$ and those who smoked $(P=0.010)$ among

125 KT patients with PAD in this cohort. The mean serum OPG level of the entire cohort was 3.08

$126 \mathrm{pg} / \mathrm{L}$. Moreover, serum OPG levels were significantly higher in patients with PAD than in those

127 without PAD (9.31 vs $2.69 \mathrm{pg} / \mathrm{L}, P=0.001)$. Immunosuppressive agents used in this cohort of

128 KT patients included tacrolimus $(n=43 ; 58.1 \%)$, mycophenolate mofetil or mycophenolic acid

$129(n=53 ; 71.6 \%)$, steroids $(n=60 ; 81.1 \%)$, rapamycin $(n=14 ; 18.9 \%)$, and cyclosporine $(n=18$;

$13024.3 \%$ ). There were no significant differences in sex, transplantation model (deceased or live

131 donor), or use of any of the immunosuppressive agents between KT patients with PAD and those 
132 without PAD.

133 Multivariate logistic regression analysis to determine whether diabetes, hypertension, or OPG

134 significantly correlated with PAD diagnosis revealed that only diabetes (odds ratio [OR], 7.120;

$13595 \%$ confidence interval [CI], 1.080-46.940; $\mathrm{P}=0.041$ ) and serum OPG level (odds ratio [OR],

$1361.336 ; 95 \%$ confidence interval $[\mathrm{CI}], 1.108-1.611 ; \mathrm{P}=0.002)$ were independent predictors of

137 PAD in KT patients after statistical adjustment of patient's characteristics as age, hypertension,

138 KT duration and eGFR (Table 3).

139 The ROC curve analysis determined that the optimal cutoff serum OPG value for predicting

140 PAD in KT patients was 7.117 pg/L (Fig. 1). Accordingly, the sensitivity, specificity, and AUC

141 of this cutoff value in predicting PAD in KT patients were $61.54 \%, 86.89 \%$, and 0.799 ,

142 respectively $(95 \% \mathrm{CI}, 0.690-0.884 ; P<0.001)$.

\section{Discussion}

145 The findings of the current study demonstrated that KT patients with PAD had higher prevalence

146 rates of diabetes and smoking and higher levels of serum OPG than those without PAD. Diabetes

147 and serum OPG were two independent clinical predictors of PAD among KT patients by

148 multivariable analysis. Cardiovascular disease is the leading cause of morbidity and mortality in

149 patients with various stages of chronic kidney disease. Moreover, the renal function of patients

150 who undergo KT unfortunately remains within the range of chronic kidney disease. Impaired

151 renal function was previously shown to predispose patients to PAD and lead to increased rates of

152 cardiovascular morbidity and mortality via multiple pathogenic mechanisms (Garimella et al.

153 2012). Consequently, central or peripheral arterial disease, which can be diagnosed by brachial-

154 ankle pulse wave velocity or $\mathrm{ABI}$, is related to renal function status and proteinuria and may 
155 contribute to the deterioration of renal function (Ohya et al. 2006; Tian et al. 2012).

156 The first evidence for a role of serum OPG in vascular calcification was derived from an

157 experimental study utilizing $O P G$ knockout mice that displayed calcification of the large arteries,

158 akin to the vascular lesions of patients with atherosclerosis (Bucay et al. 1998). The role of OPG

159 in vascular calcification depends on its act within the OPG/RANK/RANKL pathway that

160 facilitates bidirectional modulation of osteogenic, inflammatory, and apoptotic responses (Evrard

161 et al. 2015; Bernardi et al. 2016). Therefore, OPG induction by inflammatory cytokines may

162 reflect endothelial dysfunction (Van Campenhout \& Golledge 2009). Furthermore, OPG inhibits

163 vascular calcification by preventing the transformation of vascular smooth muscle cells into

164 chondrocyte-like or osteoblast-like cells in vascular tissue and by neutralizing the pro-apoptotic

165 actions of TNF-related apoptosis-inducing ligand (TRAIL) (Evrard et al. 2015). Other protective

166 roles of OPG include the inhibition of alkaline phosphatase-mediated osteogenic differentiation

167 of vascular cells and the inhibition of passive apoptotic calcification (Bucay et al. 1998; Min et

168 al. 2000). Studies conducted in animal models also demonstrated that the development of

169 vascular calcification is prevented by restoration of the $O P G$ gene. In another experimental

170 model of vascular calcification that was induced by vitamin D intoxication, OPG administration

171 prevented the formation of vascular lesions (Price et al. 2001). Studies in human exploring the

172 link between osteoporosis and vascular calcification demonstrated that low bone mineral density

173 often coincides with vascular calcification (Zhang \& Feng 2016). Relatedly, subcutaneous

174 injection of OPG inhibits osteoclastic bone resorption in postmenopausal women (Bekker et al.

175 2001). Generally, OPG plays a protective role of vascular calcification in animal studies but also

176 would be as detrimental effect on the progression of atherosclerosis in clinical consideration.

177 Increased inflammatory cytokines lead to the overproduction of OPG and may cause endothelial 
178 dysfunction (Van Campenhout \& Golledge 2009). Recent clinical studies demonstrated that

179 increased serum OPG is a significant risk factor for the progression of atherosclerosis and

180 cardiovascular disease and is positively correlated with the severity of coronary artery disease

181 (Hosbond et al. 2014; Tousoulis et al. 2013). Several clinical studies in KT patients also showed

182 that increased serum OPG levels are associated with renal and cardiovascular events as well as

183 mortality (Svensson et al. 2012; Hjelmesaeth et al. 2006). Moreover, high serum OPG levels in

184 KT patients are significantly associated with the progression and severity of abdominal aortic

185 calcification at two years after transplantation (Meneghini et al. 2013). Another study noted that

186 serum OPG is independently associated with the degree of coronary artery calcification at

187 baseline but is not at one year after KT (Bargnoux et al. 2009). Similarly, in coronary artery

188 disease, serum OPG is correlated with the severity of PAD as defined by an elevated ABI,

189 independently of the presence of diabetes, suggesting OPG as a robust marker of PAD activity

190 (O'Sullivan et al. 2010). The present study showed that serum OPG, diabetes, and smoking were

191 associated with PAD in KT patients. After adjusting for these significant factors using a stepwise

192 multivariable linear regression analysis, we found that both serum OPG and diabetes were

193 independent variables that indicated the development of PAD in KT patients in the study cohort.

194 A serum OPG value of greater than $7.577 \mathrm{pg} / \mathrm{L}$ was previously proposed to predict the presence

195 of coronary artery calcification in patients with chronic kidney disease (Morena et al. 2009). In

196 the present study, the cutoff value of serum OPG to predict the presence of PAD was $7.117 \mathrm{pg} / \mathrm{L}$,

197 whereas the AUC was 0.799. In another study on non-uremic diabetic patients, the authors found

198 that a similar cutoff level of serum OPG $(>7.371 \mathrm{pg} / \mathrm{L})$ indicated an increased risk for silent

199 myocardial ischemia independently of gender, type of diabetes, and presence of diabetic

200 nephropathy (Avignon et al. 2007). Interestingly, the cutoff values of serum OPG utilized for 
201 coronary artery disease and PAD in chronic kidney disease and KT patients were comparable,

202 even though different severity of renal function of KT patients in present study.

203 Our study has several limitations. First, this was cross-sectional study, and these findings should

204 be confirmed by long-term prospective studies before establishing a causal relationship between

205 serum OPG and PAD in KT patients. Second, the number of KT patients enrolled in the present

206 study was limited, and there were no case-matched control patients, which could potentially

207 create a selection bias. Third, the observational design of the current study did not allow us to

208 examine the mechanism underlying the statistical association between OPG and ABI observed in

209 the study. Fourth, lack of study concerned of sensitivity and specificity of ABI for diagnosis

210 PAD in KT patients, and no angiographic image was also applied in this study. Moreover, a

211 relationship between serum OPG level and inflammation in PAD was not examined in the

212 current study.

213

214 Conclusion

215 Timely detection of biological markers of vascular risk is critical in KT patients who are at an

216 increased risk for PAD. The findings of the current study demonstrated that serum OPG and

217 diabetes are positively correlated with PAD in KT patients. A cutoff serum OPG value of 7.117

$218 \mathrm{pg} / \mathrm{L}$ might be utilized to reliably predict the presence of PAD, especially in KT patients at high

219 risk of PAD, which requires aggressive clinical management.

220 


\section{References}

222 Avignon A, Sultan A, Piot C, Mariano-Goulart D, Thuan Dit Dieudonne JF, Cristol JP, and

223

224

225

226

227

228

229

230

231

232

233

234

235

236

237

238

239

240

241

242

243

Dupuy AM. 2007. Osteoprotegerin: a novel independent marker for silent myocardial ischemia in asymptomatic diabetic patients. Diabetes Care 30:2934-2939. $10.2337 / \mathrm{dc} 07-0992$

Bargnoux AS, Dupuy AM, Garrigue V, Jaussent I, Gahide G, Badiou S, Szwarc I, Deleuze S, Vernhet H, Cristol JP, and Mourad G. 2009. Evolution of coronary artery calcifications following kidney transplantation: relationship with osteoprotegerin levels. Am J Transplant 9:2571-2579. 10.1111/j.1600-6143.2009.02814.x

Bekker PJ, Holloway D, Nakanishi A, Arrighi M, Leese PT, and Dunstan CR. 2001. The effect of a single dose of osteoprotegerin in postmenopausal women. J Bone Miner Res 16:348-360. 10.1359/jbmr.2001.16.2.348

Bernardi S, Bossi F, Toffoli B, and Fabris B. 2016. Roles and Clinical Applications of OPG and TRAIL as Biomarkers in Cardiovascular Disease. Biomed Res Int 2016:1752854. $10.1155 / 2016 / 1752854$

Bucay N, Sarosi I, Dunstan CR, Morony S, Tarpley J, Capparelli C, Scully S, Tan HL, Xu W, Lacey DL, Boyle WJ, and Simonet WS. 1998. osteoprotegerin-deficient mice develop early onset osteoporosis and arterial calcification. Genes Dev 12:1260-1268.

Cooke JP, and Wilson AM. 2010. Biomarkers of peripheral arterial disease. J Am Coll Cardiol 55:2017-2023. 10.1016/j.jacc.2009.08.090

Criqui MH, and Aboyans V. 2015. Epidemiology of peripheral artery disease. Circ Res 116:1509-1526. 10.1161/circresaha.116.303849

Doobay AV, and Anand SS. 2005. Sensitivity and specificity of the ankle-brachial index to 
244

245

246

predict future cardiovascular outcomes: a systematic review. Arterioscler Thromb Vasc Biol 25:1463-1469. 10.1161/01.ATV.0000168911.78624.b7

Evrard S, Delanaye P, Kamel S, Cristol JP, and Cavalier E. 2015. Vascular calcification: from pathophysiology to biomarkers. Clin Chim Acta 438:401-414. 10.1016/j.cca.2014.08.034

Garimella PS, Hart PD, O'Hare A, DeLoach S, Herzog CA, and Hirsch AT. 2012. Peripheral artery disease and CKD: a focus on peripheral artery disease as a critical component of CKD care. Am J Kidney Dis 60:641-654. 10.1053/j.ajkd.2012.02.340

Hjelmesaeth J, Ueland T, Flyvbjerg A, Bollerslev J, Leivestad T, Jenssen T, Hansen TK, Thiel S, Sagedal S, Roislien J, and Hartmann A. 2006. Early posttransplant serum osteoprotegerin levels predict long-term (8-year) patient survival and cardiovascular death in renal transplant patients. J Am Soc Nephrol 17:1746-1754. 10.1681/asn. 2005121368

Hosbond SE, Diederichsen AC, Saaby L, Rasmussen LM, Lambrechtsen J, Munkholm H, Sand NP, Gerke O, Poulsen TS, and Mickley H. 2014. Can osteoprotegerin be used to identify the presence and severity of coronary artery disease in different clinical settings? Atherosclerosis 236:230-236. 10.1016/j.atherosclerosis.2014.07.013

Hsu BG, Shih MH, Chen YC, Ho GJ, Lin TY, and Lee MC. 2015. High Serum Osteoprotegerin Is Associated with Arterial Stiffness in Kidney Transplant Patients. Tohoku J Exp Med 236:247-253. 10.1620/tjem.236.247

Lee CJ, Wang JH, Chen ML, Yang CF, Chen YC, and Hsu BG. 2015. Serum osteoprotegerin is associated with arterial stiffness assessed according to the cardio-ankle vascular index in hypertensive patients. J Atheroscler Thromb 22:304-312. 10.5551/jat.25882 
267 Lee MC, Chen YC, Ho GJ, Shih MH, Chou KC, and Hsu BG. 2014. Serum leptin levels

268 positively correlate with peripheral arterial stiffness in kidney transplantation patients. Transplant Proc 46:353-358. 10.1016/j.transproceed.2013.11.145

270

271

272

273

274

275

276

277

278

279

280

281

282

283

284

285

286

287

288

289

Chronic Kidney Disease Epidermiology Collaboration. 2006. Using standardized serum creatinine values in the modification of diet in renal disease study equation for estimating glomerular filtration rate. Ann Intern Med 15;145:247-54.

Meneghini M, Regalia A, Alfieri C, Barretta F, Croci D, Gandolfo MT, Vettoretti S, Rastaldi MP, and Messa P. 2013. Calcium and osteoprotegerin levels predict the progression of the abdominal aortic calcifications after kidney transplantation. Transplantation 96:4248. 10.1097/TP.0b013e3182934cee

Min H, Morony S, Sarosi I, Dunstan CR, Capparelli C, Scully S, Van G, Kaufman S, Kostenuik PJ, Lacey DL, Boyle WJ, and Simonet WS. 2000. Osteoprotegerin reverses osteoporosis by inhibiting endosteal osteoclasts and prevents vascular calcification by blocking a process resembling osteoclastogenesis. J Exp Med 192:463-474.

Morena M, Dupuy AM, Jaussent I, Vernhet H, Gahide G, Klouche K, Bargnoux AS, Delcourt C, Canaud B, and Cristol JP. 2009. A cut-off value of plasma osteoprotegerin level may predict the presence of coronary artery calcifications in chronic kidney disease patients. Nephrol Dial Transplant 24:3389-3397. 10.1093/ndt/gfp301

O'Sullivan EP, Ashley DT, Davenport C, Kelly J, Devlin N, Crowley R, Leahy AL, Kelly CJ, Agha A, Thompson CJ, O'Gorman DJ, Fitzgerald P, and Smith D. 2010.

Osteoprotegerin is higher in peripheral arterial disease regardless of glycaemic status. Thromb Res 126:e423-427. 10.1016/j.thromres.2010.09.003 
290 Ohya Y, Iseki K, Iseki C, Miyagi T, Kinjo K, and Takishita S. 2006. Increased pulse wave 291 velocity is associated with low creatinine clearance and proteinuria in a screened 292 cohort. Am J Kidney Dis 47:790-797. 10.1053/j.ajkd.2006.01.027

293 Price PA, June HH, Buckley JR, and Williamson MK. 2001. Osteoprotegerin inhibits artery 294 calcification induced by warfarin and by vitamin D. Arterioscler Thromb Vasc Biol 21:1610-1616.

296

297

298

299

300 301

302

303

304

305

306

307

308

309

310

311

312

Fellstrom B, and Holdaas H. 2012. Osteoprotegerin as a predictor of renal and cardiovascular outcomes in renal transplant recipients: follow-up data from the ALERT study. Nephrol Dial Transplant 27:2571-2575. 10.1093/ndt/gfr694

Tian SL, Tian XK, Han QF, Axelsson J, and Wang T. 2012. Presence of peripheral arterial disease predicts loss of residual renal function in incident CAPD patients. Perit Dial Int 32:67-72. 10.3747/pdi.2010.00109

Tousoulis D, Siasos G, Maniatis K, Oikonomou E, Kioufis S, Zaromitidou M, Paraskevopoulos T, Michalea S, Kollia C, Miliou A, Kokkou E, Papavassiliou AG, and Stefanadis C. 2013. Serum osteoprotegerin and osteopontin levels are associated with arterial stiffness and the presence and severity of coronary artery disease. Int J Cardiol 167:1924-1928. 10.1016/j.ijcard.2012.05.001

Van Campenhout A, and Golledge J. 2009. Osteoprotegerin, vascular calcification and atherosclerosis. Atherosclerosis 204:321-329. 10.1016/j.atherosclerosis.2008.09.033

Venuraju SM, Yerramasu A, Corder R, and Lahiri A. 2010. Osteoprotegerin as a predictor of coronary artery disease and cardiovascular mortality and morbidity. J Am Coll Cardiol 55:2049-2061. 10.1016/j.jacc.2010.03.013 
313 Wang JH, Lee CJ, Chen ML, Yang CF, Chen YC, and Hsu BG. 2014. Association of serum

314 osteoprotegerin levels with carotid-femoral pulse wave velocity in hypertensive

315 patients. J Clin Hypertens (Greenwich) 16:301-308. 10.1111/jch.12288

316 Ye Z, Ali Z, Klee GG, Mosley TH, Jr., and Kullo IJ. 2013. Associations of candidate biomarkers

317 of vascular disease with the ankle-brachial index and peripheral arterial disease. Am J

$318 \quad$ Hypertens 26:495-502. 10.1093/ajh/hps073

319 Zhang Y, and Feng B. 2016. Systematic review and meta-analysis for the association of bone

320 mineral density and osteoporosis/osteopenia with vascular calcification in women. Int

$321 \quad$ J Rheum Dis. 10.1111/1756-185x.12842 


\section{Table $\mathbf{1}$ (on next page)}

Baseline parameters of kidney transplantation patients with or without peripheral artery disease.

Continuous variables are presented as means \pm standard deviation and tested by Student's $t$ test. Variables that are not normally distributed are presented as medians with interquartile range and tested using the Mann-Whitney U test.

eGFR, estimated glomerular filtration rate; KT, kidney transplantation; HDL-C, high-density lipoprotein cholesterol; LDL-C, low-density lipoprotein cholesterol.

$* \mathrm{P}<0.05$ indicating statistical significance 
1 Table 1. Baseline parameters of kidney transplantation patients with or without peripheral artery

2 disease.

\begin{tabular}{|c|c|c|c|c|}
\hline Parameter & $\begin{array}{l}\text { All patients } \\
\qquad(n=74)\end{array}$ & $\begin{array}{c}\text { No peripheral artery } \\
\text { disease group }(n=61)\end{array}$ & $\begin{array}{c}\text { Peripheral artery } \\
\text { disease group }(n=13)\end{array}$ & $P$ value \\
\hline Age (years) & $52.07 \pm 9.63$ & $51.87 \pm 9.12$ & $53.00 \pm 12.15$ & 0.703 \\
\hline $\begin{array}{l}\text { Post-KT duration } \\
\text { (months) }\end{array}$ & $72.19 \pm 42.99$ & $69.51 \pm 40.97$ & $84.77 \pm 51.42$ & 0.248 \\
\hline Height (cm) & $162.16 \pm 8.33$ & $162.56 \pm 8.15$ & $160.31 \pm 9.24$ & 0.380 \\
\hline Body weight (kg) & $62.61 \pm 12.59$ & $62.46 \pm 11.66$ & $63.31 \pm 16.84$ & 0.827 \\
\hline $\begin{array}{l}\text { Waist circumference } \\
(\mathrm{cm})\end{array}$ & $85.12 \pm 11.41$ & $85.00 \pm 11.07$ & $85.69 \pm 13.39$ & 0.844 \\
\hline $\begin{array}{l}\text { Body mass index } \\
\left(\mathrm{kg} / \mathrm{m}^{2}\right)\end{array}$ & $23.74 \pm 4.21$ & $23.59 \pm 4.05$ & $24.43 \pm 5.05$ & 0.522 \\
\hline $\begin{array}{l}\text { Left ankle-brachial } \\
\text { index }\end{array}$ & $1.07 \pm 0.15$ & $1.11 \pm 0.12$ & $0.88 \pm 0.16$ & $<0.001 *$ \\
\hline $\begin{array}{l}\text { Right ankle-brachial } \\
\text { index }\end{array}$ & $1.06 \pm 0.14$ & $1.10 \pm 0.10$ & $0.88 \pm 0.13$ & $<0.001 *$ \\
\hline $\begin{array}{l}\text { Systolic blood pressure } \\
(\mathrm{mmHg})\end{array}$ & $139.05 \pm 16.57$ & $138.26 \pm 15.11$ & $142.77 \pm 22.61$ & 0.377 \\
\hline $\begin{array}{l}\text { Diastolic blood pressure } \\
(\mathrm{mmHg})\end{array}$ & $86.18 \pm 10.88$ & $87.07 \pm 10.43$ & $82.00 \pm 12.34$ & 0.128 \\
\hline Albumin (mg/dL) & $4.12 \pm 0.50$ & $4.16 \pm 0.46$ & $3.93 \pm 0.467$ & 0.136 \\
\hline Globulin (mg/dL) & $2.82 \pm 0.59$ & $2.802 \pm 0.62$ & $2.862 \pm 0.47$ & 0.992 \\
\hline $\begin{array}{l}\text { Total cholesterol } \\
(\mathrm{mg} / \mathrm{dL})\end{array}$ & $195.79 \pm 45.84$ & $197.87 \pm 47.88$ & $186.00 \pm 34.60$ & 0.400 \\
\hline Triglyceride (mg/dL) & $114.50(80.75-167.00)$ & $117.00(80.50-166.50)$ & $85.00(71.00-170.00)$ & 0.491 \\
\hline HDL-C (mg/dL) & $51.34 \pm 15.93$ & $50.52 \pm 14.55$ & $55.15 \pm 21.57$ & 0.345 \\
\hline
\end{tabular}




\begin{tabular}{lcccc}
\hline LDL-C (mg/dL) & $108.79 \pm 38.97$ & $108.07 \pm 35.00$ & $112.15 \pm 55.68$ & 0.734 \\
\hline Fasting glucose (mg/dL) & $93.50(86.00-110.00)$ & $95.00(88.00-111.00)$ & $92.00(80.50-97.00)$ & 0.164 \\
\hline $\begin{array}{l}\text { Blood urea nitrogen } \\
(\mathrm{mg} / \mathrm{dL})\end{array}$ & $22.50(17.00-34.25)$ & $23.00(17.50-30.50)$ & $19.00(15.50-48.00)$ & 0.881 \\
\hline Creatinine (mg/dL) & $1.60(1.28-2.10)$ & $1.58(1.30-2.10)$ & $1.90(1.05-2.35)$ & 0.771 \\
\hline eGFR (mL/min) & $42.80 \pm 22.25$ & $42.87 \pm 21.84$ & $42.46 \pm 25.02$ & 0.953 \\
\hline $\begin{array}{lccc}\text { Total calcium (mg/dL) } \\
9.19 \pm 1.04\end{array}$ & $9.22 \pm 1.10$ & $9.04 \pm 0.72$ & 0.569 \\
\hline Phosphorus (mg/dL) & $3.42 \pm 0.86$ & $3.36 \pm 0.88$ & $3.66 \pm 0.74$ & 0.261 \\
\hline $\begin{array}{l}\text { Intact parathyroid } \\
\text { hormone (pg/mL) }\end{array}$ & $111.65(63.63-160.73)$ & $117.20(74.15-162.65)$ & $75.20(45.65-150.25)$ & 0.196 \\
\hline Osteoprotegerin (pg/L) & $3.08(1.27-6.85)$ & $2.69(1.22-5.46)$ & $9.31(3.46-14.13)$ & $0.001^{*}$ \\
\hline
\end{tabular}

3

4 Continuous variables are presented as means \pm standard deviation and tested by Student's $t$ test.

5 Variables that are not normally distributed are presented as medians with interquartile range and

6 tested using the Mann-Whitney $U$ test.

7 eGFR, estimated glomerular filtration rate; KT, kidney transplantation; HDL-C, high-density

8 lipoprotein cholesterol; LDL-C, low-density lipoprotein cholesterol.

$9 * P<0.05$ indicating statistical significance 


\section{Table 2 (on next page)}

Clinical characteristics of kidney transplantation patients with or without peripheral artery disease.

Data are expressed as number of patients, and analyses are performed using the chi-square test.

$* P<0.05$ indicating statistical significance 
1 Table 2. Clinical characteristics of kidney transplantation patients with or without peripheral

2 artery disease.

\begin{tabular}{|c|c|c|c|c|}
\hline Characteristic & & $\begin{array}{l}\text { No peripheral artery } \\
\text { disease group }(\%)\end{array}$ & $\begin{array}{l}\text { Peripheral artery } \\
\text { disease group }(\%)\end{array}$ & $P$ value \\
\hline \multirow{2}{*}{ Gender } & Male & $34(55.7)$ & $6(46.2)$ & \multirow{2}{*}{0.529} \\
\hline & Female & $27(44.3)$ & $7(53.8)$ & \\
\hline \multirow{2}{*}{ Diabetes } & No & $47(77.0)$ & $6(46.2)$ & \multirow{2}{*}{$0.025^{*}$} \\
\hline & Yes & $14(23.0)$ & $7(53.8)$ & \\
\hline \multirow{2}{*}{ Hypertension } & No & $31(50.8)$ & $7(53.8)$ & \multirow{2}{*}{0.843} \\
\hline & Yes & $30(49.2)$ & $6(46.2)$ & \\
\hline \multirow[t]{2}{*}{ Smoking } & No & $59(96.7)$ & $10(76.9)$ & \multirow[t]{2}{*}{$0.010 *$} \\
\hline & Yes & $2(3.3)$ & $3(23.1)$ & \\
\hline \multirow{2}{*}{ Transplantation model } & Deceased donor & $53(86.9)$ & $12(92.3)$ & \multirow{2}{*}{0.587} \\
\hline & Living donor & $8(13.1)$ & $1(7.7)$ & \\
\hline \multirow{2}{*}{ Tacrolimus use } & No & $26(42.6)$ & $5(38.5)$ & \multirow{2}{*}{0.782} \\
\hline & Yes & $35(57.4)$ & $8(61.5)$ & \\
\hline \multirow{2}{*}{$\begin{array}{l}\text { Mycophenolate mofetil or } \\
\text { mycophenolic acid use }\end{array}$} & No & $15(24.6)$ & $6(46.2)$ & \multirow{2}{*}{0.117} \\
\hline & Yes & $46(75.4)$ & $7(53.8)$ & \\
\hline \multirow{2}{*}{ Steroid use } & No & $12(19.7)$ & $2(15.4)$ & \multirow{2}{*}{0.720} \\
\hline & Yes & $49(80.3)$ & $11(84.6)$ & \\
\hline \multirow{2}{*}{ Rapamycin use } & No & $50(82.0)$ & $10(76.9)$ & \multirow{2}{*}{0.673} \\
\hline & Yes & $11(18.0)$ & $3(23.1)$ & \\
\hline \multirow{2}{*}{ Cyclosporine use } & No & $45(73.8)$ & $11(84.6)$ & \multirow{2}{*}{0.408} \\
\hline & Yes & $16(26.2)$ & $2(15.4)$ & \\
\hline
\end{tabular}

3

4 Data are expressed as number of patients, and analyses are performed using the chi-square test.

$5 * P<0.05$ indicating statistical significance 


\section{Table 3(on next page)}

Multivariate logistic regression analysis to determine factors correlated with peripheral arterial disease among kidney transplantation patients $(n=74)$. 
1 Table 3. Odds ratio for peripheral arterial disease by multivariate logistic regression analysis

2 among the 74 kidney transplantation patients.

\begin{tabular}{|c|c|c|c|c|c|c|}
\hline \multirow[t]{2}{*}{ Variables } & \multicolumn{2}{|c|}{ Model 1} & \multicolumn{2}{|c|}{ Model 2} & \multicolumn{2}{|c|}{ Model 3} \\
\hline & OR $(95 \% \mathrm{CI})$ & $P$-value & OR $(95 \% \mathrm{CI})$ & $P$-value & OR $(95 \% \mathrm{CI})$ & $P$-value \\
\hline Osteoprotegerin (pg/L) & $\begin{array}{c}1.297(1.102- \\
1.527)\end{array}$ & $0.002 *$ & $\begin{array}{c}1.305(1.101- \\
1.546)\end{array}$ & $0.002^{*}$ & $\begin{array}{c}1.336(1.108- \\
1.611)\end{array}$ & $0.002 *$ \\
\hline Diabetes mellitus & $\begin{array}{c}4.846 \\
(1.041-22.550)\end{array}$ & $0.044^{*}$ & $\begin{array}{c}6.729 \\
(1.151-39.328)\end{array}$ & $0.034^{*}$ & $\begin{array}{c}7.120 \\
(1.080-46.940)\end{array}$ & $0.041 *$ \\
\hline
\end{tabular}

4 Model 1 is adjusted for diabetes mellitus, smoking, and osteoprotegerin.

5 Model 2 is adjusted for the Model 1 variables and for age and hypertension.

6 Model 3 is adjusted for the Model 2 variables and for kidney transplantation duration and

7 glomerular filtration rate.

$8 * P<0.05$ by multivariate logistic regression analysis.

9 CI, confidence interval; OR, odds ratio. 
Figure 1

ROC curve for optimal cutoff value of serum OPG

Receiver operating characteristic (ROC) curve determined that the optimal cutoff value of serum osteoprotegerin for predicting arterial stiffness in patients with kidney transplantation was $7.117 \mathrm{pg} / \mathrm{L}$. The area under the ROC curve (AUC) for osteoprotegerin was 0.799 (95\% confidence interval, $0.690-0.884 ; \mathrm{P}<0.001$ ), with a sensitivity of $61.54 \%$ and a specificity of $86.89 \%$.

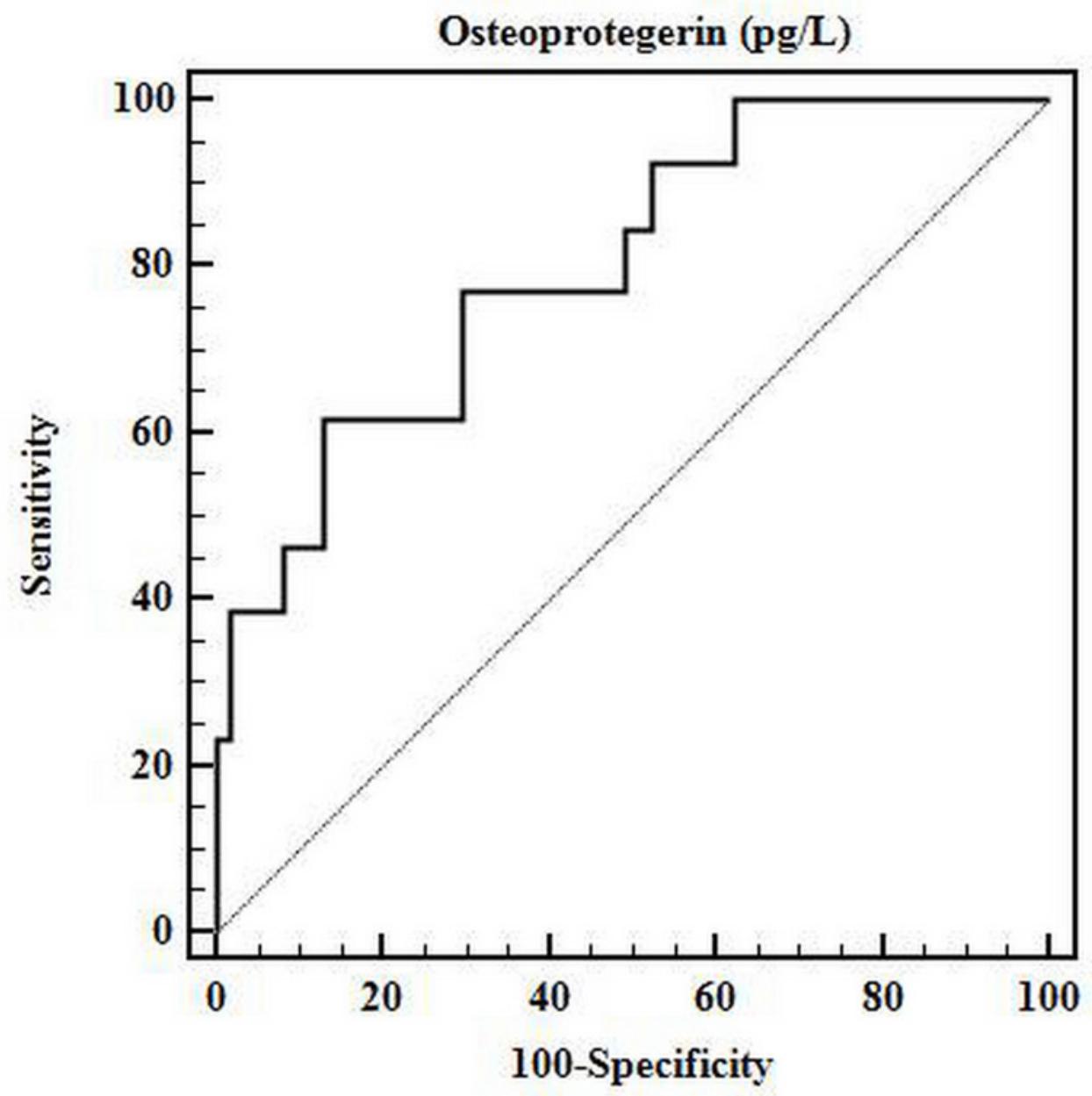

